\title{
ATP synthase is required for male fertility and germ cell maturation in Drosophila testes
}

\author{
JUN YU ${ }^{1-3^{*}}$, BINGHAI CHEN ${ }^{4 *}$, BO ZHENG $^{2,5^{*}}$, CHEN QIAO $^{6^{*}}$, XIA CHEN $^{1 *}$, \\ YIDAN YAN ${ }^{1}$, XIAOJIN LUAN ${ }^{1}$, BING XIE $^{1}$, JIAJIA LIU ${ }^{1}$, CONG SHEN $^{5}$, ZEYU HE $^{7}$, \\ XING HU ${ }^{1}$, MINGXI LIU ${ }^{2}, \mathrm{HONG} \mathrm{LI}^{5}$, QIXIANG SHAO ${ }^{3,8}$ and JIE FANG ${ }^{1}$ \\ ${ }^{1}$ Department of Gynecology, Affiliated Hospital of Jiangsu University, Zhenjiang, Jiangsu 212001; \\ ${ }^{2}$ Department of Histology and Embryology, State Key Laboratory of Reproductive Medicine, Nanjing Medical University, \\ Nanjing, Jiangsu 211166; ${ }^{3}$ Reproductive Sciences Institute of Jiangsu University; ${ }^{4}$ Department of Urology, \\ Affiliated Hospital of Jiangsu University, Zhenjiang, Jiangsu 212001; ${ }^{5}$ Center for Reproduction and Genetics, \\ Suzhou Municipal Hospital, Nanjing Medical University Affiliated Suzhou Hospital, Suzhou, Jiangsu 215002; \\ ${ }^{6}$ Department of Clinical Pharmacy, Affiliated Hospital of Jiangsu University, Zhenjiang, Jiangsu 212001; \\ ${ }^{7}$ Department of Clinical Medicine, China Medical University, Shenyang, Liaoning 110001; \\ ${ }^{8}$ Department of Immunology, Jiangsu Key Laboratory of Medical Science and Laboratory Medicine, \\ School of Medicine, Jiangsu University, Zhenjiang, Jiangsu 212013, P.R. China
}

Received March 26, 2018; Accepted November 8, 2018

DOI: $10.3892 / \mathrm{mmr} .2019 .9834$

\begin{abstract}
Germ cell maturation is essential for spermatogenesis and testis homeostasis. ATP synthase serves significant roles in energy storage in germ cell survival and is catalyzed by alterations in the mitochondrial membrane proton concentration. The intrinsic cellular mechanisms governing stem cell maturation remain largely unknown. In the present study, in vivo RNA interference (RNAi) screening of major ATP synthase subunits was performed, and the function of ATP synthase for male fertility and spermatogenesis in Drosophila was explored. A Upstream Activation Sequence/Gal4 transcription factor system was used to knock down gene expression in specific cell types, and immunofluorescence staining was conducted to assess the roles of ATP synthase subunits in Drosophila testes. It was identified that knockdown of ATP synthase resulted in male infertility and abnormal spermatogenesis in Drosophila testes. In addition, knockdown of the ATP synthase $\beta$ subunit in germ cells resulted in defects in male infertility and germ cell maturation, while the hub and cyst cell populations were maintained. Other major ATP
\end{abstract}

Correspondence to: Dr Jun Yu or Professor Jie Fang, Department of Gynecology, Affiliated Hospital of Jiangsu University, 438 Jiefang Road, Zhenjiang, Jiangsu 212001, P.R. China

E-mail: yujun9117@126.com

E-mail: fangjie070@163.com

${ }^{*}$ Contributed equally

Key words: adenosine 5'-triphosphate synthase, male fertility, germ cell mature, Drosophila, testis synthase subunits were also examined and similar phenotypes in Drosophila testes were identified. Taken together, the data from the present study revealed that ATP synthase serves important roles for male fertility during spermatogenesis by regulating germ cell maturation in Drosophila testes.

\section{Introduction}

Drosophila is an excellent model organism for the study of male fertility and spermatogenesis (1-3). Drosophila has a short life cycle, distinct development stages, and a streamlined genome with high levels of gene conservation, with certain genes similar to those in humans (3). Furthermore, various genetic tools and reagent resources for complex biological processes are available in Drosophila $(4,5)$.

The process of spermatogenesis is conserved between Drosophila and humans $(6,7)$. A number of genes including Boule homolog, RNA binding protein and Deleted in azoospermia have been identified to be involved in male fertility, sharing similar phenotypes in Drosophila and humans $(8,9)$. Spermatogenesis in the adult Drosophila testis involves germline stem cells, spermatogonia, spermatocytes, spermatids and sperm (10). At the tip of the Drosophila testis, a stem cell niche controls the maintenance and differentiation of germline stem cells (GSCs) and cyst stem cells (CySCs) by somatic hub cells. GSCs divide to generate two cell types: A new stem cell and a gonialblast that proliferates and differentiates into spermatocytes $(7,11)$. Cyst cells provide a microenvironment for germ cell proliferation, growth and differentiation (6).

Adenosine 5'-triphosphate (ATP) synthase is an enzyme complex that creates the energy storage molecule ATP, which is commonly used to provide energy for the majority of tissues and organisms $(12,13)$. Mitochondrial ATP synthase has been 
identified in the mitochondrial inner membrane and is involved in oxidative phosphorylation, catalyzing ATP synthesis via a proton $(\mathrm{H}+)$ gradient $(14,15)$. Mitochondrial defects due to dysfunctional ATP synthase complexes in humans have been demonstrated to cause neuromuscular disorders (16). The ATP synthase complex typically contains at least 15 subunits [a, A6L, b, c, d, e, f, g, oligomycin sensitivity-conferring protein (OSCP), f6, $\alpha, \beta, \gamma, \delta$ and $\varepsilon]$ that are divided among two regions, F1 and F0. The F1 portion extends into the mitochondrial matrix, physically attaching in two ways to the F0 portion, which is embedded in the inner membrane, via the F1 central stalk that allows rotation, and via the peripheral or stator stalk, which stabilizes the complex (17).

In Drosophila, ATP is generated by two main approaches: Glycolysis; and mitochondrial respiration. Mitochondrial respiration is the most efficient source of ATP; therefore, the ATP required for the growth of germ cells is primarily obtained through mitochondrial respiration. Notably, certain studies indicated that ATP synthase subunits were required for mitochondrial morphogenesis and germ cell development $(18,19)$. An additional study suggested that inhibiting ATP synthase activity suppresses boar sperm motility (20). Mitochondrial ferritin (FtMt), which is a highly expressed protein in the testis, is a functional ferritin binding to mitochondria. FtMt contributes to sperm epididymis maturation and to male fertility, and the cauda epididymides of $\mathrm{FtMt}^{-/}$mice have been identified to exhibit decreased fertility (21). A previous study on Drosophila ovaries demonstrated that ATP synthase promoted the maturation of mitochondrial cristae during differentiation through dimerization and specific upregulation of the ATP synthase complex (22). However, the regulatory function of other ATP synthase subunits in spermatogenesis in the Drosophila testis remains unclear.

The Upstream Activation Sequence/Gal4 transcription factor (UAS/Gal4) system-based RNA interference (RNAi) silencing method has previously been used to analyze the male biological reproductive process in Drosophila (23). Different Gal4 drivers exhibit different expression levels and patterns. The present study examined the role of ATP synthase subunits in mediating the maturation and cell fate of late-stage germ cells within the Drosophila testes. A total of 2 Gal4 stocks were used to drive UAS-ATPsyn RNAi expression in Drosophila testis. Nanos-Gal4 (Nos-Gal4) has germ cell-specific expression, primarily in early stage germ cells $(24,25)$. The expression of bag of marbles-Gal4 (bam-Gal4) is restricted to transit-amplifying spermatogonia (TA-spermatogonia) $(25,26)$. The results suggested that ATP synthase is required for male fertility and serves important roles in maintaining germ cell differentiation and growth in Drosophila testes; these data may provide novel insights for the etiological diagnosis of male infertility.

\section{Materials and methods}

Fly strains. All flies were fed with standard corn meal food at $25^{\circ} \mathrm{C}$. Information about the alleles and transgenes used in the present study are available either in FlyBase (www.flybase. org) or as stated: Nos-Gal4 (4937; Bloomington Drosophila Stock Center, Dept Biology, Indiana University, Bloomington, IN, USA), bam-Gal4; $\Delta 86 /+$ was a gift from Professor Dahua
Chen (Institute of Zoology, Chinese Academy of Sciences, Beijing, China) and has been described in a previous study (11). All UAS-RNAi transgenic fly lines were obtained from The TsingHua Fly Center (THFC; Beijing, China). Drosophila melanogaster Canton-Special flies were used as the wild type (WT) strain.

Fly crosses and male fertility test. Fly crosses and male fertility tests were performed as described in our previous study (3). Transgenic UAS-RNAi males were crossed with virgin females carrying nos-Gal4 or bam-Gal4 drivers at $25^{\circ} \mathrm{C}$. The testis phenotype was analyzed by using F1 RNAi adult male flies for immunofluorescence staining. Single male fertility tests were performed using a single F1 RNAi adult male flies enclosed for 3 days with three WT virgin females at room temperature. All F1 RNAi adult male flies were analyzed at $48 \mathrm{~h}$ after hatching.

Immunofluorescence and antibodies. Fly testes were dissected in $1 \mathrm{X}$ PBS and fixed at room temperature for $30 \mathrm{~min}$ in $4 \%$ paraformaldehyde. Following washing three times in $1 \mathrm{X}$ PBS with $0.1 \%$ Triton X-100 (PBST) and blocking at room temperature for $1 \mathrm{~h}$ in $5 \%$ bovine serum albumin (Sangon Biotech, Shanghai, China) dissolved in 1XPBS, the samples were incubated with primary antibodies overnight at $4^{\circ} \mathrm{C}$. Following washing three times for $10 \mathrm{~min}$ in $0.1 \%$ PBST, the samples were then incubated for $1 \mathrm{~h}$ with secondary antibodies (Alexa Fluor ${ }^{\circledR} 488$ or $\mathrm{Cy}^{\mathrm{TM}}$ 3) at room temperature followed by three washes in $0.1 \%$ PBST. Testes were then stained with Hoechst 33342 (1.0 mg/ml; Invitrogen; Thermo Fisher Scientific, Inc, Waltham, MA, USA) at room temperature for 5 min prior to mounting.

The antibodies used were as follows: Mouse anti-EYA transcriptional coactivator and phosphatase 1 [Eya; 1:20; eya10H6; Developmental Studies Hybridoma Bank (DSHB), Iowa City, IA, USA]; rat anti-Drosophila E-cadherin homolog (DE-cadherin; 1:20; DCAD2; DSHB); rabbit anti-DEAD-Box helicase 4 (Vasa; 1:1000; cat. no. sc-30210; Santa Cruz Biotechnology, Inc., Dallas, TX, USA). Secondary antibodies conjugated to Alexa Fluor ${ }^{\circledR}$ 488-rabbit (cat. no. 711-545-152; Jackson ImmunoResearch Laboratories, Inc., West Grove, PA, USA) or Cy ${ }^{\mathrm{TM}}$ 3-mouse (cat. no. 715-165-150; Jackson ImmunoResearch Laboratories, Inc.) were diluted at 1:1,000.

Bioinformatics analysis. Diagrams indicating the oxidative phosphorylation process and distribution of the subunits of ATP synthase were adopted from the Kyoto Encyclopedia of Genes and Genomes Database (http://www.kegg.jp). Gene ontology (GO) analysis was performed, and clusters were analyzed using the Database For Annotation, Visualization, and Integrated Discovery (DAVID) Bioinformatics Database (https://david.ncifcrf.gov/) $(27,28)$. The data were evaluated for statistical differences using the Benjamini-Hochberg method.

Statistical analysis. Experiments were repeated at least three times. The fertility rate was evaluated for statistical differences using one-way analysis of variance and Least Significance Difference post-hoc test by SPSS software (v22; IBM Corp., Armonk, NY, USA). P<0.05 was considered to indicate a statistically significant difference. 

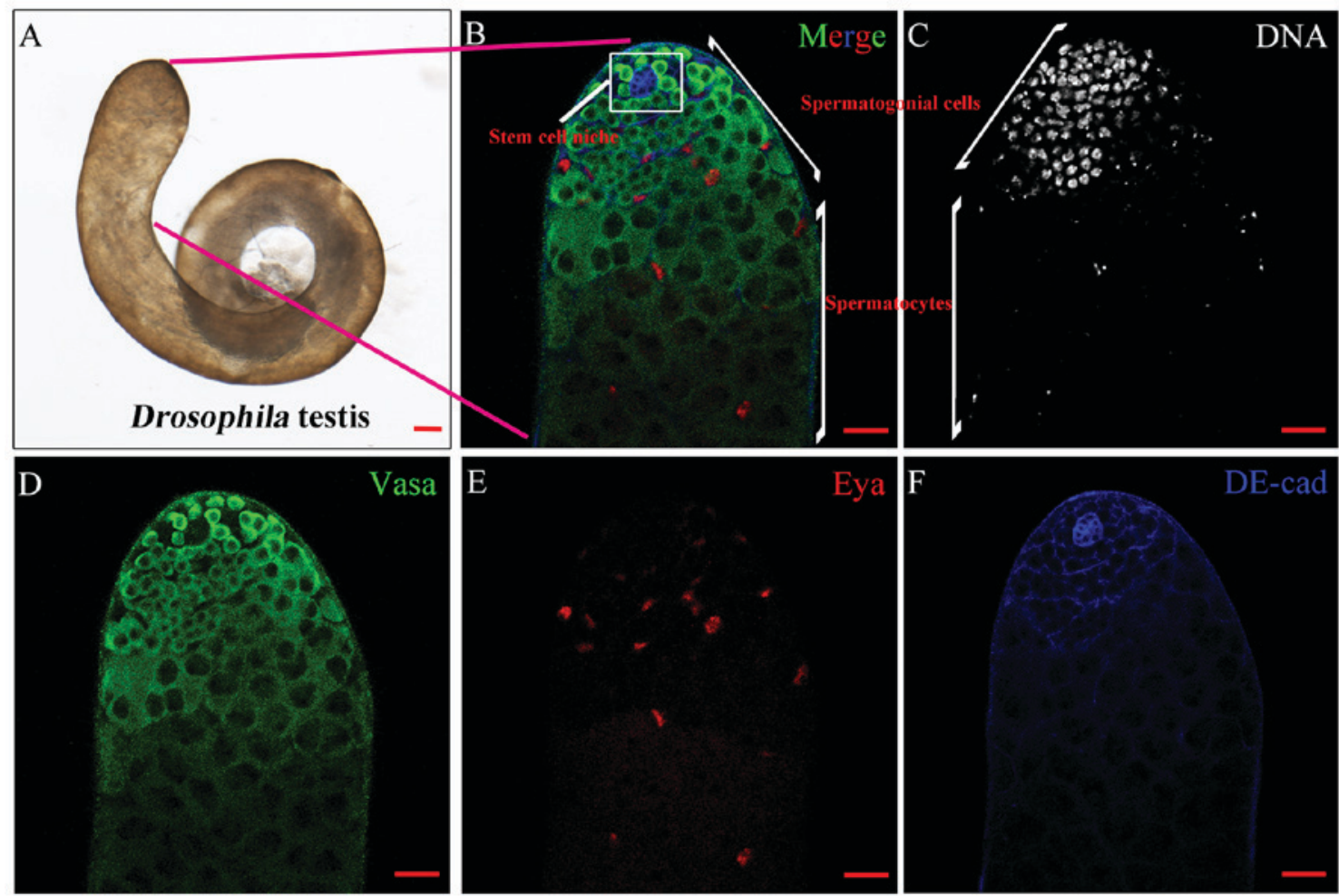

Figure 1. Structure of the WT testis in Drosophila. (A) Whole mount of the wild type testis by light microscope. Scale bar, $100 \mu$ m. (B) Complete immunofluorescence image of the tip of the testis with stem cell niche, spermatogonial cells and spermatocytes, as labelled. Scale bar, $20 \mu \mathrm{m}$. (C) DNA (grey) staining highlighted the area of spermatogonial cells. Scale bar, $20 \mu \mathrm{m}$. (D) Vasa (green) and (E) Eya (red) stained cells represented germ and cyst cells, respectively. Scale bars, $20 \mu \mathrm{m}$. (F) Hub cells and cyst cells were stained by DE-cadherin (blue). Scale bar, $20 \mu \mathrm{m}$. Vasa, DEAD-Box helicase 4; Eya, EYA transcriptional coactivator and phosphatase 1 .

\section{Results}

Structure of the Drosophila testis. Drosophila has been demonstrated to be an excellent model organism in which to screen genes essential for male fertility. Adult Drosophila testes contain spermatogonia, spermatocytes, spermatids and sperm. In the present study, a whole mount of WT testis was captured by light microscope (Fig. 1A). At the apical tip of the Drosophila testis (Fig. 1B), the stem cell niche in the Drosophila testis maintained the self-renewal and differentiation capabilities of GSCs and CySCs. The results demonstrated that spermatogonial cells, but not spermatocytes, were extensively stained by Hoechst 33342 DNA dye (Fig. 1C). Immunofluorescence staining with Vasa, Eya and DE-cad was able to identify germ cells, cyst cells, and somatic hub and cyst cells, respectively (Fig. 1D-F).

ATP synthase $\beta$ subunit is required for male fertility. To examine the function of the ATP synthase $\beta$ subunit in Drosophila testes, two independent ATP synthase $\beta$ RNAi lines (ATPsyn $\beta$ RNAi-1 and ATPsyn $\beta$ RNAi-2) were selected to evaluate the male fertility rate. In the nos>UAS-ATPsyn $\beta$ RNAi flies (Fig. 2A), numerous males [69.66\% $(n=89)$ for ATPsyn $\beta$ RNAi-1; $53.85 \%(n=78)$ for ATPsyn $\beta$ RNAi-2] were infertile compared with WT flies $(3.30 \%$; $n=91)$. When bam-Gal4 was used to knock down ATPsyn $\beta$ expression in 2- to 16-cell spermatogonia (Fig. 2B), it was identified that a proportion of the resulting males [32.32\% $(n=99)$ for ATPsyn $\beta$ RNAi-1; $22.86 \%(n=105)$ for ATPsyn $\beta$ RNAi-2] were infertile. The results suggested that ATP synthase $\beta$ was essential for male fertility.

ATP synthase $\beta$ subunit is essential for germ cell maturation. To additionally explore the role of the ATP synthase $\beta$ subunit, the structure of the testis and changes in the expression pattern were detected at the molecular level by immunofluorescence. The Eya gene is required for the development of the Drosophila compound eyes and organ morphogenesis $(29,30)$. A previous study has indicated that Eya is a protein with nuclear localization that is expressed in mature cyst cells in Drosophila testes (31). Eya was used as a positive marker for cyst cells in the present study. Vasa usually functions as a common marker label for germ cells.

Knockdown of the ATP synthase $\beta$ subunit in early germ cells resulted in a significant decrease in Vasa-positive germ cells in spermatocytes in nos>ATPsyn $\beta$ RNAi testes [59.10\% of testes with defects in ATPsyn $\beta$ RNAi-1 $(n=22) ; 61.90 \%$ of testes with defects in ATPsyn $\beta$ RNAi-2 $(n=21)]$ compared with WT testes $(n=32)$, but there was no significant difference in the number of GSCs and spermatogonia between the knockdown and WT testes (Fig. 3A-C). Despite the dysfunction in germ cell maturation, hub and cyst cells were not affected. These data suggest that loss of the ATP synthase $\beta$ subunit may cause defects in the later stages of germ cell maturation, but not affect the early stages of spermatogenesis and somatic cells.

To additionally evaluate the biological function of the ATP synthase $\beta$ subunit, ATPsyn $\beta$ was knocked down using 


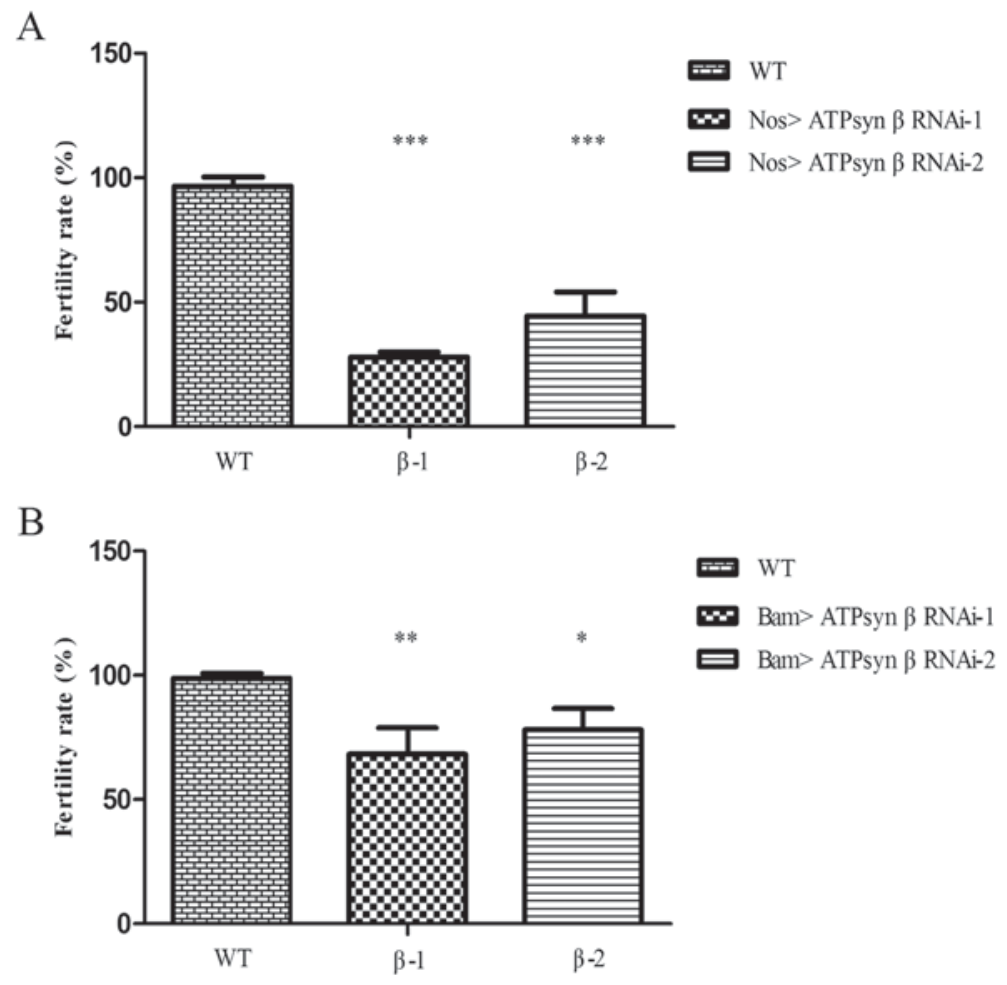

Figure 2. Fertility rate of flies with ATP synthase $\beta$ subunit knockdown. A total of two independent RNAi lines of ATPsyn $\beta$ (ATPsyn $\beta$ RNAi-1 and ATPsyn $\beta$ RNAi-2) were used. (A) The fertility rate of WT and nos>ATPsyn $\beta$ RNAi males. (B) The fertility rate of WT and bam>ATPsyn $\beta$ RNAi males. Data were evaluated by one-way analysis of variance and Least Significant Difference post-hoc test. ${ }^{*} \mathrm{P}<0.05,{ }^{* *} \mathrm{P}<0.01$ and ${ }^{* * *} \mathrm{P}<0.001$. WT, wild type; ATP, adenosine 5'-triphosphate; RNAi, RNA interference; ATPsyn, ATP synthase; Nos, Nanos; Bam, bag of marbles.
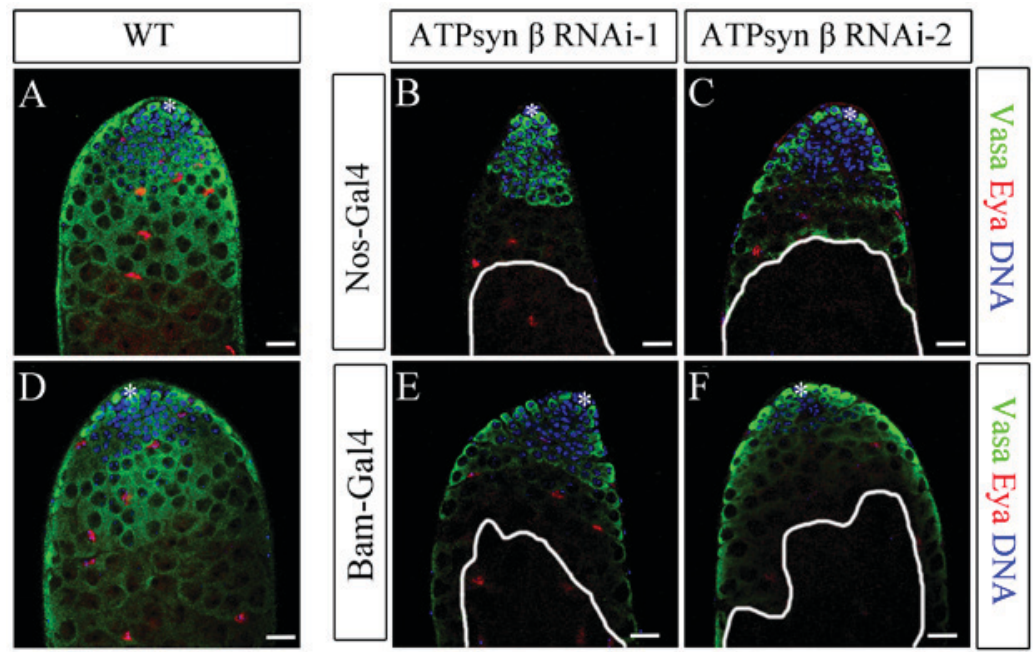

Figure 3. Knockdown of ATP synthase $\beta$ subunit with nos-Gal4 and bam-Ga14. (A-C) Immunofluorescence of (A) WT, (B) nos $>$ ATPsyn $\beta$ RNAi-1 and (C) nos $>$ ATPsyn $\beta$ RNAi-2 testes. (D-F) Immunofluorescence of (D) WT, (E) bam>ATPsyn $\beta$ RNAi-1 and (F) bam>ATPsyn $\beta$ RNAi-2 testes. Representative images of WT testes illustrate the tip of the testis with hub cells, GSCs, CySCs, differentiated germ cells and mature cyst cells. Germ, cyst, and undifferentiated germ cells were stained to detect Vasa (green stain), Eya (red stain), and DNA (blue stain). Areas enclosed by white lines represent regions of germ cells with maintenance defects. Scale bars, $20 \mu \mathrm{m}$ WT, wild type; ATP, adenosine 5'-triphosphate; ATPsyn, ATP synthase; Nos, Nanos; Bam, bag of marbles; RNAi, RNA interference; ", hub cells; Vasa, DEAD-Box helicase 4; Eya, EYA transcriptional coactivator and phosphatase 1.

bam-Gal4. The Bam protein is a key differentiation factor in early germ cells, and it determines the differentiation fate of spermatogonia and triggers spermatocyte development $(32,33)$. In bam>ATPsyn $\beta$ RNAi testes $[47.83 \%$ of testes with defects in ATPsyn $\beta$ RNAi-1 $(n=23) ; 55.17 \%$ of testes with defects in ATPsyn $\beta$ RNAi-2 $(n=29)]$, the spermatocytes partially disappeared, whereas the hub and cyst cells remained (Fig. 3D-F). Knockdown of ATPsyn $\beta$ driven by bam-Gal4 was identified to exhibit a similar phenotype of germ cell maturation defects.

Knockdown of major ATP synthase subunits causes partial male infertility in Drosophila testes. In the present study, the function of ATP synthase in Drosophila testis was 
Table I. ATPsyn RNA interference strains used in the screening process.

\begin{tabular}{llccccc}
\hline ATPsyn & THFC no. & BDSC no. & TRiP no. & Annotation symbol & Gene symbol & Hairpin ID \\
\hline $\mathrm{b}$ & THU2903 & 28062 & JF02899 & CG8189 & ATPsynB & TR02373P.1 \\
$\mathrm{c}$ & THU0360 & 35464 & GL00390 & CG1746 & ATPsynC & SH01517.N2 \\
$\mathrm{d}$ & THU1424 & 33740 & HMS01078 & CG6030 & ATPsynD & SH01691.N \\
OSCP & TH01379.N2 & - & - & CG4307 & ATPsynO & SH03719.N2 \\
f6 & TH01666.N & - & - & CG4412 & ATPsynCF6 & SH04247.N \\
$\alpha$ & THU2900 & 28059 & JF02896 & CG3612 & ATPsyn- $\alpha$ & TR02362P.1 \\
$\beta-1$ & THU2798 & 27712 & JF02792 & CG11154 & ATPsyn-beta & TR02374P.1 \\
$\beta-2$ & THU2896 & 28056 & JF02892 & CG11154 & ATPsyn-beta & TR02347P.1 \\
$\gamma$ & THU3092 & 28723 & JF03150 & CG7610 & ATPsyn-gamma & TR02371P.1 \\
\hline
\end{tabular}

ATP, adenosine 5'-triphosphate; THFC, TsingHua Fly Center; BDSC, Bloomington Drosophila Stock Center; OSCP, oligomycin sensitivity-conferring protein; TRiP, The Transgenic RNAi Project.
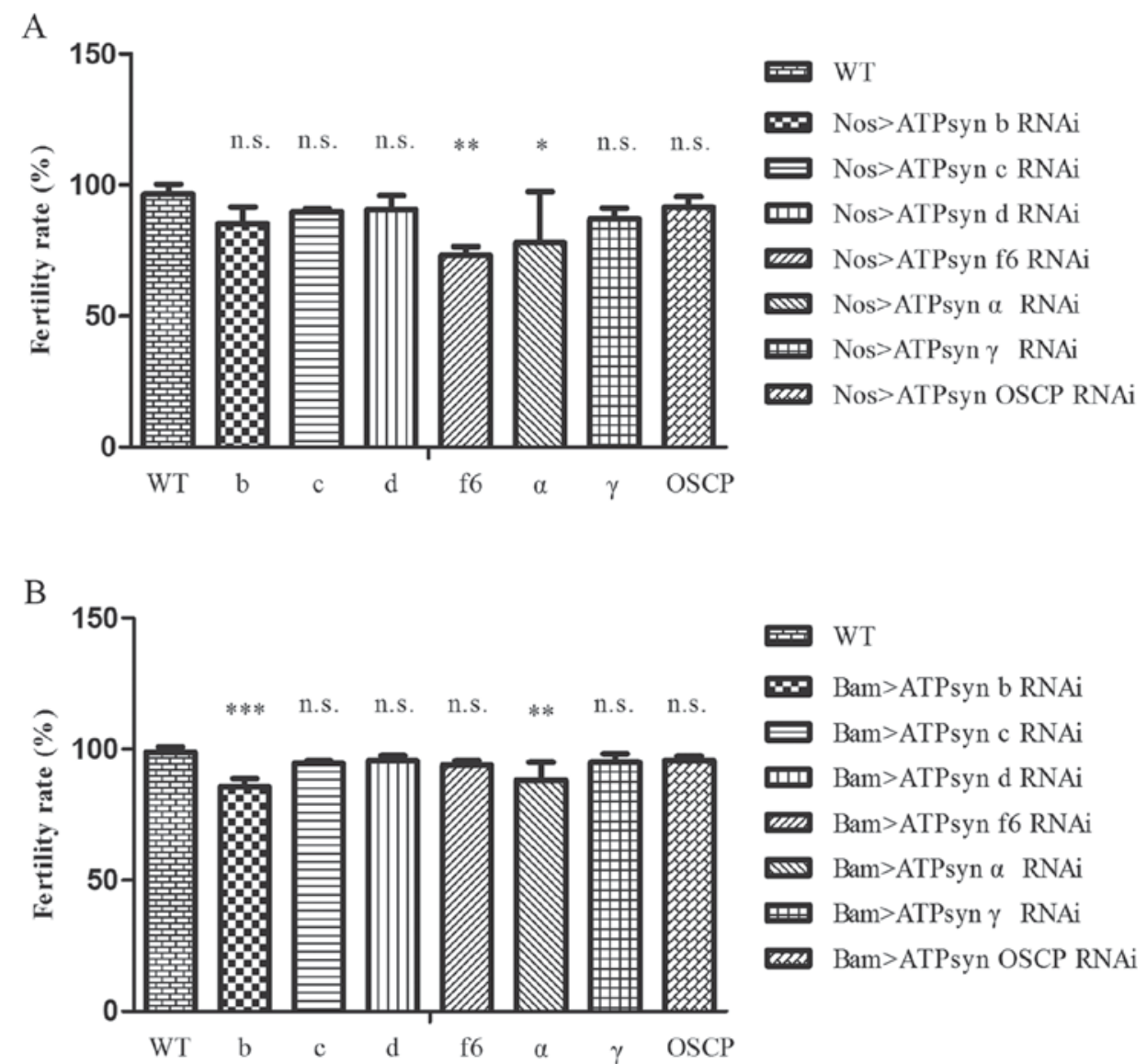

Figure 4. Fertility rate of knockdown of major ATP synthase subunits. (A) The fertility rate of WT and major ATP synthase subunit RNAi knockout flies driven by nos-Gal4. (B) The fertility rate of WT and major ATP synthase subunit RNAi knockouts driven by bam-Gal4. Data were evaluated by one-way analysis of variance and Least Significant Difference post-hoc test. ${ }^{*} \mathrm{P}<0.05,{ }^{* *} \mathrm{P}<0.01$ and ${ }^{* * *} \mathrm{P}<0.001$. ATP, adenosine 5 '-triphosphate; ATPsyn, ATP synthase; Nos, Nanos; Bam, bag of marbles; WT, wild type; RNAi, RNA interference; OSCP, oligomycin sensitivity-conferring protein; n.s., no statistical difference.

systematically analyzed. A total of 2 independent ATPsyn $\beta$ RNAi lines were used and defects in germ cell maturation in testes whose RNAi knockdown was driven by the combination of nos-Gal4 and bam-Gal4 were also identified, indicating that the ATPsyn $\beta$ subunit serves a key role in germ cell fate. However, the function of other major ATP synthase subunits remained uncharacterized.
To detect whether other ATP synthase subunits also served significant roles in male fertility and germ cell mature in testes, an in vivo RNAi screening assay was conducted using a USA-Gal4 system. To additionally explore the function of ATP synthase in testes, RNAi lines of ATP synthase subunits from THFC were selected (Table I). The RNAi lines from the THFC were from the same RNAi collection as the Transgenic 


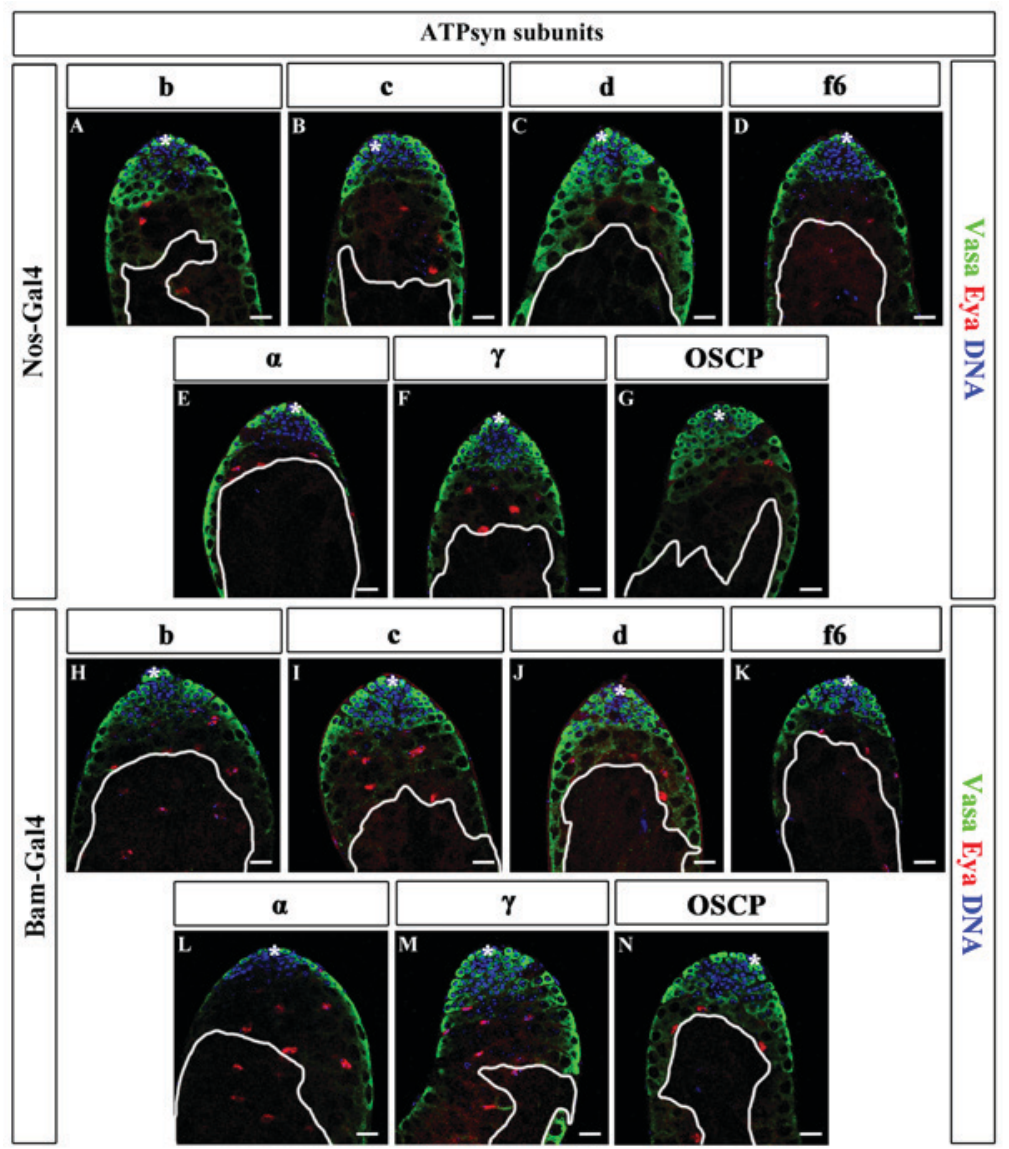

Figure 5. Knockdown of major ATP synthase subunits in germ cells. (A-G) Immunofluorescence of ATPsyn RNAi testes driven by nos-Gal4, including (A) b, (B) c, (C) d, (D) f6, (E) $\alpha$, (F) $\gamma$ and (G) OSCP. (H-N) Immunofluorescence of ATPsyn subunits in RNAi testes driven by bam-Gal4, including (H) b, (I) c, (J) d, (K) f6, (L) $\alpha,\left(\right.$ M) $\gamma$ and (N) OSCP. Areas enclosed by white lines represent regions of germ cells with maintenance defects. Scale bars, $20 \mu \mathrm{m} .{ }^{*}$, hub cells; ATP, adenosine 5'-triphosphate; ATPsyn, ATP synthase; Nos, Nanos; Bam, bag of marbles; WT, wild type; RNAi, RNA interference; OSCP, oligomycin sensitivity-conferring protein; Vasa, DEAD-Box helicase 4; Eya, EYA transcriptional coactivator and phosphatase 1.

RNAi Project (34). Finally, the function of 8 of the $15(53.3 \%)$ major ATP synthase subunits, namely, ATPsyn b, ATPsyn c, ATPsyn d, ATPsyn OSCP, ATPsyn f6, ATPsyn $\alpha$, ATPsyn $\beta$, and ATPsyn $\gamma$ were screened for.

For the male fertility test, it was identified that the fertility levels of the ATPsyn f6 RNAi and ATPsyn $\alpha$ RNAi strains, driven by nos-Gal4, were partially affected (Fig. 4A; $\mathrm{P}<0.05$ ). In addition, males with ATPsyn b RNAi and ATPsyn $\alpha$ RNAi, driven by bam-Gal4. exhibited a decrease in the fertility rate (Fig. 4B; $\mathrm{P}<0.05$ ). These results demonstrated that defects in sections of the ATP synthase subunits may affect male fertility in Drosophila testes.

Knockdown of major ATP synthase subunits results in germ cell maturation defects in Drosophila testes. When nos-Gal4 was used for screening, all the lines exhibited germ cell maturation defects. These testes were stained with the germ cell marker Vasa. In the majority of the ATPsyn RNAi testes, early germ cells, including GSCs and TA-spermatogonia, were Vasa-positive. In addition, certain spermatocytes in a number of ATPsyn RNAi testes were Vasa-negative (8/15 in ATPsyn b RNAi; 9/17 in ATPsyn c RNAi; 6/19 in ATPsyn d RNAi, 10/25 in ATPsyn OSCP RNAi; 11/19 in ATPsyn f6 RNAi; $15 / 25$ in ATPsyn $\alpha$ RNAi; and 13/27 in ATPsyn $\gamma$ RNAi). ATPsyn RNAi testes were also stained with the somatic cyst cell marker Eya, and all cells were identified to be positive for Eya (Fig. 5A-G). The results suggest that ATP synthase does not affect the survival of GSCs and TA-spermatogonia. However, ATP synthase may be vital for germ cell maturation.

Next, the ability of germ cell maturation in ATP synthase-deficient testes, driven by bam-Gal4, was examined. Similar germ cell maturation defects were identified in bam>ATPsyn RNAi testes: In $48.00 \%$ (12/25) ATPsyn b RNAi, 29.17\% (7/24) ATPsyn c RNAi, 29.63\% (8/27) ATPsyn d RNAi, 44.00\% (11/25) ATPsyn OSCP RNAi, 42.11\% (8/19) ATPsyn f6 RNAi, 31.82\% (7/22) ATPsyn $\alpha$ RNAi and $47.80 \%$ (11/23) ATPsyn $\gamma$ RNAi testes, some of the spermatocytes were Vasa-negative, while the early germ cells and somatic hub and cyst cells were not affected (Fig. 5H-N). These data indicate that the major ATP synthase subunits are key factors for germ cell maturation.

ATP synthase Assembly and GO analysis. The roles of most of the major ATP synthase subunits remain to be elucidated. Analysis of the major ATP synthase subunits driven by nos-Gal4 and bam-Gal4 indicated that ATP synthase subunits may assemble into a complex and participate in germ cell development via oxidative phosphorylation (Fig. 6A). In the present study, 8 of the 15 major ATP synthase subunits were examined, and were identified as being required 


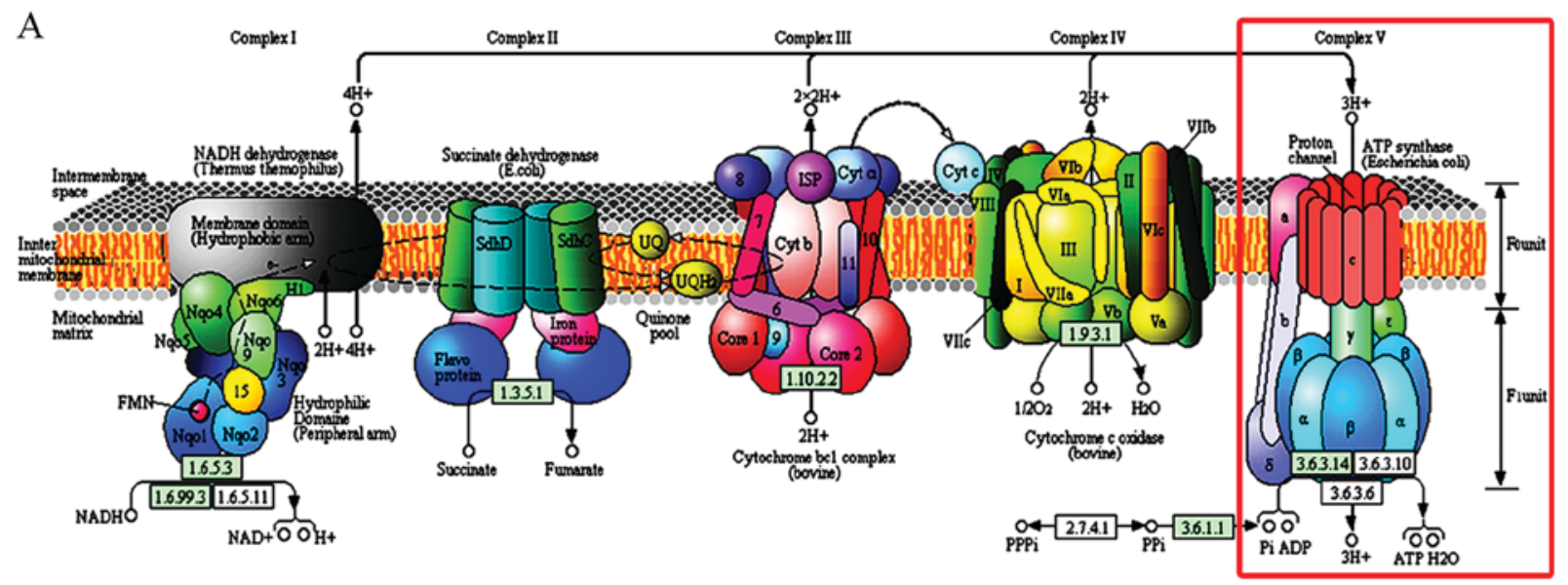

B

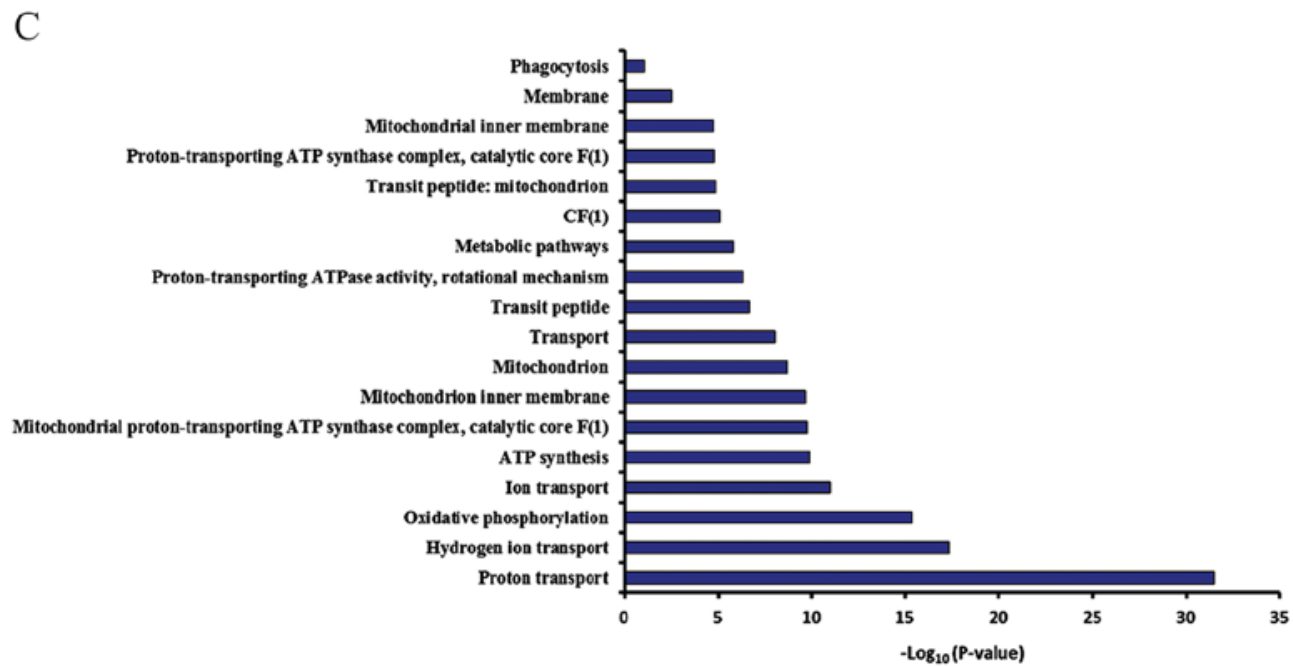

Figure 6. Assembly of ATP synthase and GO analysis of ATP synthase subunits. (A) A Kyoto Encyclopedia of Genes and Genomes pathway map indicates that ATP synthase assembles into a complex and is involved in the development of germ cells by oxidative phosphorylation. Electron transport through complexes I-IV drives the extrusion of protons into the intermembrane space. The proton gradient generated is harnessed by the ATP synthase to drive the synthesis of ATP. (B) The colored boxes indicate which ATP synthase components were screened. (C) Clusters identified by the Database For Annotation, Visualization, and Integrated Discovery bioinformatics database indicate selected GO terms of genes identified in the ATP synthase screening process. ATP, adenosine 5'-triphosphate; GO, Gene Ontology.

for the maturation of germ cells during the later stages (Fig. 6B). Cellular and cell cycle components required for basic metabolism are generally thought to be expressed at consistent levels throughout the development process. The present study additionally conducted GO analysis of major ATP synthase subunits using DAVID. The list of the top functional annotations of ATP synthase contained oxidative phosphorylation, proton transport, mitochondrion, and ATP synthesis $(\mathrm{P}<0.05$; Fig. 6C). The results from the present study revealed that the mitochondrial ATP synthase serves an important role in promoting the developmentally regulated maturation of germ cells in Drosophila testis.

\section{Discussion}

The Drosophila testis provides an excellent model to study spermatogenesis. Mitochondrial ATP synthase catalyzes ATP synthesis and produces energy for germ cell development. Mitochondria are widely expressed in germ cells and serve key roles in male fertility and germ cell survival $(35,36)$.
At present, only a few studies have analyzed the regulatory network of ATP synthase in the testis $(19,37,38)$. The present study, using Drosophila as an in vivo model, systematically analyzed a series of ATP synthase subunits and explored their common regulation of germ cell maturation in the Drosophila testis. Notably, it was identified that ATPsyn $\beta$ is a regulatory factor in germ cell maturation. Wen et al (39) demonstrated that knockout of hpRNA1 inhibited its target ATPsyn $\beta$ in testes, and affected spermatogenesis and male fertility. Notably, hpRNA1 mutant seminal vesicles exhibited a lower density of sperm in the accessory gland and mild defection of nuclear organization in the testis tail. The present study primarily focused on male fertility and germ cell maturation. Knockdown of ATPsyn $\beta$ resulted in partial infertility and caused germ cell maturation defects. It was also observed that a small proportion of germ cells were able to undergo the meiosis process and form sperm, which was consistent with the data from Wen et al (39).

A previous study indicated that the ATP synthase served to promote the maturation of mitochondrial cristae during 
differentiation through dimerization and specific upregulation of the ATP synthase complex in Drosophila ovaries (22). Proliferating cysts in ATP synthase knockdown models failed to continue to differentiate and were unable to progress from four- to eight-cell cysts in Drosophila ovaries, and they demonstrated that mitochondrial ATP synthase serves a critical role in stem cell differentiation process (22). Despite the fact that germline stem cells are strictly controlled by stem cell niches in both Drosophila testes and ovaries, gametogenesis in Drosophila testes is quite different from that in ovaries. The results from the present study demonstrated that knockdown of ATP synthase subunits did not result in germ cell differentiation defects, but affected germ cell maintenance in Drosophila testes, indicating its critical role in spermatogenesis.

A previous study has demonstrated that the knotted onions (knon) gene encodes a testis-specific paralog of ATP synthase subunit $d$, and is required for the internal structure of the nebenkern and its subsequent disassembly and elongation. knon knockout mutants exhibited aberrant mitochondrial elongation during spermatogenesis and faulty nebenkern morphology (19). Knockdown of the ATP synthase subunits f6 and g in Drosophila testis revealed a phenotype similar to that of knon mutants (19). In addition, ATP synthase subunit $\mathrm{b}$ has been demonstrated to be essential for the growth, development and male fertility in Caenorhabditis elegans (40). Deficiency of the ATP synthase subunit $\mathrm{b}$ in testes was identified to disrupt nuclear bundles during spermatogenesis and cause abnormal shaping and spermatid elongation (40). Chen et al (41) demonstrated that ubiquitous knockdown of ATP synthase subunit $b$ resulted in growth defects, and knockdown of ATP synthase subunit $\mathrm{b}$ in testes caused infertility and abnormal spermatogenesis, which was consistent with the data from the present study. Nevertheless, Chen et al (41) only used one testis-associated Gal4 for the knockdown of ATP synthase subunit b, and primarily focused on the staining of testicle tail in Drosophila. The present study additionally examined the male fertility and testicular apex staining by two different germ cell-associated Gal4s (nos-Gal4 and bam-Gal4), and several markers to distinguish different cell types were also used; it was identified that spermatocytes were Vasa-negative in knockdown testes.

The present study described evidence associating germ cell mature and cell survival to ATP synthase. The data demonstrated the role of a cell biological process in germ cell development, and these results contribute to the knowledge of the role of ATP synthase in ATP production.

In a number of cell types, dimerization of ATP synthase complexes with their axes at a certain angle is important for determining the sharp positive curvature of the inner mitochondrial membrane $(19,42)$. It was hypothesized that deficiency of ATP synthase subunits in Drosophila testis may alter ATP synthase dimerization or affect the assembly and stability of the ATP synthase complex in the inner mitochondrial membrane, blocking the delivery of energy, which is essential for germ cell development. However, knockdown of ATP synthase subunits in early germ cells did not affect the survival of GSCs. It was hypothesized that GSCs in Drosophila testes may obtain their energy through glycolysis or the noncanonical approach.
Future studies on ATP synthase subunits and other members of the oxidative phosphorylation system may explore their regulatory network and mechanism underlying the clustered regularly interspersed short palindromic repeats (CRISPR) associated protein 9/CRISPR system in Drosophila. Future studies should also examine the mutation rate of ATP synthase subunits in patients with oligoasthenospermia. The data from the present study may assist to reveal the mechanisms underlying male infertility and oligoasthenospermia.

\section{Acknowledgements}

The authors would like to thank Professor Dahua Chen from Institute of Zoology, Chinese Academy of Sciences for sharing reagents and stocks.

\section{Funding}

The present study was supported by National Natural Science Foundation of China (grant nos. 31701298 and 81402100), Natural Science Foundation of Jiangsu Province (grant no. BK20170562), Key Research Foundation of Zhenjiang Social Development (grant nos. SH2017013, SH2017020, SH2016028, SH2016031 and SH2014026), Key Research Foundation of Zhenjiang Health Science and Technology (grant no. SHW2016001), Open Fund of State Key Laboratory of Reproductive Medicine of Nanjing Medical University (grant no. SKLRM-KA201603), the Foundation of Health and Family Planning Commission of Jiangsu Province (grant no. Q201408), the Foundation for Young Medical Talents of Jiangsu province (grant no. QNRC2016840), Six Talent Peaks Project in Jiangsu Province (grant no. WSW-007), Science Foundation of Doctorate Research of Affiliated Hospital of Jiangsu University (grant no. jdfyRC2016005), Suzhou Key Medical Center (grant no. SZZX201505), Suzhou Introduced Project of Clinical Medical Expert Team (grant no. SZYJTD201708) and Jiangsu Provincial Medical Innovation Team (grant no. CXTDB2017013).

\section{Availability of data and materials}

All data generated or analyzed during this study are included in this published article.

\section{Authors' contributions}

JY, BC and JF conceived and designed the experiments. BZ, $\mathrm{CQ}, \mathrm{XC}, \mathrm{YY}$ and $\mathrm{XL}$ performed the experiments. BX, ZH and JL analyzed the data. CS, XH and QS contributed to the fly feeding and part of the data analysis. ML and HL contributed to the fly crosses and male fertility test. HL, JF and JY wrote the manuscript. All authors read and approved the final manuscript.

\section{Ethics approval and consent to participate}

The present study was approved by Ethics Committee for Biomedical Research at Affiliated Hospital of Jiangsu University. 


\section{Patient consent for publication}

Not applicable.

\section{Competing interests}

The authors declare that they have no competing interests.

\section{References}

1. Hu Z, Li Z, Yu J, Tong C, Lin Y, Guo X, Lu F, Dong J, Xia Y, Wen Y, et al: Association analysis identifies new risk loci for non-obstructive azoospermia in Chinese men. Nat Commun 5: 3857,2014

2. Yu J, Liu Y, Lan X, Wu H, Wen Y, Zhou Z, Hu Z, Sha J, Guo X and Tong C: CHES-1-like, the ortholog of a non-obstructive azoospermia-associated gene, blocks germline stem cell differentiation by upregulating Dpp expression in Drosophila testis. Oncotarget 7: 42303-42313, 2016.

3. Yu J, Wu H, Wen Y, Liu Y, Zhou T, Ni B, Lin Y, Dong J, Zhou Z, $\mathrm{Hu}$ Z, et al: Identification of seven genes essential for male fertility through a genome-wide association study of non-obstructive azoospermia and RNA interference-mediated large-scale functional screening in Drosophila. Hum Mol Genet 24: 1493-1503, 2015.

4. Wu H, Sun L, Wen Y, Liu Y, Yu J, Mao F, Wang Y, Tong C, Guo X, Hu Z, et al: Major spliceosome defects cause male infertility and are associated with nonobstructive azoospermia in humans. Proc Natl Acad Sci USA 113: 4134-4139, 2016.

5. Hackstein JH, Hochstenbach R and Pearson PL: Towards an understanding of the genetics of human male infertility: Lessons from flies. Trends Genet 16: 565-572, 2000.

6. de Cuevas M and Matunis EL: The stem cell niche: Lessons from the Drosophila testis. Development 138: 2861-2869, 2011.

7. Spradling A, Fuller MT, Braun RE and Yoshida S: Germline stem cells. Cold Spring Harb Perspect Biol 3: a002642, 2011

8. Fuller MT and Spradling AC: Male and female Drosophila germline stem cells: Two versions of immortality. Science 316 402-404, 2007.

9. Xu EY, Lee DF, Klebes A, Turek PJ, Kornberg TB and Reijo Pera RA: Human BOULE gene rescues meiotic defects in infertile flies. Hum Mol Genet 12: 169-175, 2003.

10. White-Cooper H: Studying how flies make sperm-investigating gene function in Drosophila testes. Mol Cell Endocrinol 306 : 66-74, 2009.

11. Yu J, Lan X, Chen X, Yu C, Xu Y, Liu Y, Xu L, Fan HY and Tong C: Protein synthesis and degradation are essential to regulate germline stem cell homeostasis in Drosophila testes. Development 143: 2930-2945, 2016.

12. He J, Ford HC, Carroll J, Ding S, Fearnley IM and Walker J: Persistence of the mitochondrial permeability transition in the absence of subunit $\mathrm{c}$ of human ATP synthase. Proc Natl Acad Sci USA 114: 3409-3414, 2017.

13. Junge W and Nelson N: ATP synthase. Annu Rev Biochem 84 631-657, 2015.

14. Walker JE: The ATP synthase: The understood, the uncertain and the unknown. Biochem Soc Trans 41: 1-16, 2013.

15. Mitchell P: Chemiosmotic coupling in oxidative and photosynthetic phosphorylation. 1966. Biochim Biophys Acta 1807: 1507-1538, 2011.

16. Kucharczyk R, Zick M, Bietenhader M, Rak M, Couplan E, Blondel M, Caubet SD and di Rago JP: Mitochondrial ATP synthase disorders: Molecular mechanisms and the quest for curative therapeutic approaches. Biochim Biophys Acta 1793: 186-199, 2009.

17. Velours J, Paumard P, Soubannier V, Spannagel C, Vaillier J, Arselin $\mathrm{G}$ and Graves PV: Organisation of the yeast ATP synthase $\mathrm{F}(0)$ : A study based on cysteine mutants, thiol modification and cross-linking reagents. Biochim Biophys Acta 1458: 443-456, 2000.

18. Hendriks WK, Colleoni S, Galli C, Paris DB, Colenbrander B, Roelen BA and Stout TA: Maternal age and in vitro culture affect mitochondrial number and function in equine oocytes and embryos. Reprod Fertil Dev 27: 957-968, 2015.
19. Sawyer EM, Brunner EC, Hwang Y, Ivey LE, Brown O, Bannon M, Akrobetu D, Sheaffer KE, Morgan O, Field CO, et al: Testis-specific ATP synthase peripheral stalk subunits required for tissue-specific mitochondrial morphogenesis in Drosophila. BMC Cell Biol 18: 16, 2017.

20. Ramió-Lluch L, Yeste M, Fernández-Novell JM, Estrada E, Rocha L, Cebrián-Pérez JA, Muiño-Blanco T, Concha II, Ramírez A and Rodríguez-Gil JE: Oligomycin A-induced inhibition of mitochondrial ATP-synthase activity suppresses boar sperm motility and in vitro capacitation achievement without modifying overall sperm energy levels. Reprod Fertil Dev 26: 883-897, 2014.

21. Maccarinelli F, Regoni M, Carmona F, Poli M, Meyron-Holtz EG and Arosio P: Mitochondrial ferritin deficiency reduces male fertility in mice. Reprod Fertil Dev 29: 2005-2010, 2017.

22. Teixeira FK, Sanchez CG, Hurd TR, Seifert JR, Czech B, Preall JB, Hannon GJ and Lehmann R: ATP synthase promotes germ cell differentiation independent of oxidative phosphorylation. Nat Cell Biol 17: 689-696, 2015.

23. Ni JQ, Zhou R, Czech B, Liu LP, Holderbaum L, Yang-Zhou D, Shim HS, Tao R, Handler D, Karpowicz P, et al: A genome-scale shRNA resource for transgenic RNAi in Drosophila. Nat Methods 8: 405-407, 2011.

24. Liu M, Lim TM and Cai Y: The Drosophila female germline stem cell lineage acts to spatially restrict DPP function within the niche. Sci Signal 3: ra57, 2010.

25. White-Cooper H: Tissue, cell type and stage-specific ectopic gene expression and RNAi induction in the Drosophila testis. Spermatogenesis 2: 11-22, 2012.

26. Singh SR, Zhen W, Zheng Z, Wang H, Oh SW, Liu W, Zbar B, Schmidt LS and Hou SX: The Drosophila homolog of the human tumor suppressor gene BHD interacts with the JAK-STAT and Dpp signaling pathways in regulating male germline stem cell maintenance. Oncogene 25: 5933-5941, 2006.

27. Huang da W, Sherman BT and Lempicki RA: Systematic and integrative analysis of large gene lists using DAVID bioinformatics resources. Nat Protoc 4: 44-57, 2009.

28. Huang da W, Sherman BT and Lempicki RA: Bioinformatics enrichment tools: Paths toward the comprehensive functional analysis of large gene lists. Nucleic Acids Res 37: 1-13, 2009.

29. Furuya M, Qadota H, Chisholm AD and Sugimoto A: The C. elegans eyes absent ortholog EYA-1 is required for tissue differentiation and plays partially redundant roles with PAX-6. Dev Biol 286: 452-463, 2005.

30. Karandikar UC, Jin M, Jusiak B, Kwak S, Chen R and Mardon G: Drosophila eyes absent is required for normal cone and pigment cell development. PLoS One 9: e102143, 2014.

31. Fabrizio JJ, Boyle M and DiNardo S: A somatic role for eyes absent (eya) and sine oculis (so) in Drosophila spermatocyte development. Dev Biol 258: 117-128, 2003.

32. Bunt SM and Hime GR: Ectopic activation of Dpp signalling in the male Drosophila germline inhibits germ cell differentiation. Genesis 39: 84-93, 2004.

33. Shivdasani AA and Ingham PW: Regulation of stem cell maintenance and transit amplifying cell proliferation by tgf-beta signaling in Drosophila spermatogenesis. Curr Biol 13: 2065-2072, 2003.

34. Yan D, Neumüller RA, Buckner M, Ayers K, Li H, Hu Y, Yang-Zhou D, Pan L, Wang X, Kelley C, et al: A regulatory network of Drosophila germline stem cell self-renewal. Dev Cell 28: 459-473, 2014.

35. Chen JV and Megraw TL: Spermitin: A novel mitochondrial protein in Drosophila spermatids. PLoS One 9: e108802, 2014.

36. Wu CH, Zong Q, Du AL, Zhang W, Yao HC, Yu XQ and Wang YF: Knockdown of Dynamitin in testes significantly decreased male fertility in Drosophila melanogaster. Dev Biol 420: 79-89, 2016

37. Collins CM, Malacrida B, Burke C, Kiely PA and Dunleavy EM: ATP synthase F1 subunits recruited to centromeres by CENP-A are required for male meiosis. Nat Commun 9: 2702, 2018.

38. Midzak AS, Chen H, Aon MA, Papadopoulos V and Zirkin BR: ATP synthesis, mitochondrial function, and steroid biosynthesis in rodent primary and tumor Leydig cells. Biol Reprod 84: 976-985, 2011.

39. Wen J, Duan H, Bejarano F, Okamura K, Fabian L, Brill JA, Bortolamiol-Becet D, Martin R, Ruby JG and Lai EC: Adaptive regulation of testis gene expression and control of male fertility by the Drosophila hairpin RNA pathway. Mol Cell 57: 165-178, 2015. 
40. Kawasaki I, Hanazawa M, Gengyo-Ando K, Mitani S, Maruyama I and Iino Y: ASB-1, a germline-specific isoform of mitochondrial ATP synthase b subunit, is required to maintain the rate of germline development in Caenorhabditis elegans. Mech Dev 124: 237-251, 2007.

41. Chen YN, Wu CH, Zheng Y, Li JJ, Wang JL and Wang YF: Knockdown of ATPsyn-b caused larval growth defect and male infertility in Drosophila. Arch Insect Biochem Physiol 88 144-54, 2015.

42. Davies KM, Anselmi C, Wittig I, Faraldo-Gómez JD and Kühlbrandt W: Structure of the yeast F1FO-ATP synthase dimer and its role in shaping the mitochondrial cristae. Proc Natl Acad Sci USA 109: 13602-13607, 2012.

cc) (i) $(-)$ This work is licensed under a Creative Commons

$$
\text { Attribution-NonCommercial-NoDerivatives } 4.0
$$
International (CC BY-NC-ND 4.0) License. 